\title{
Flow Characteristics of Plane Jet from an Asymmetrical Two-Dimensional Nozzle
}

\author{
Katsuya HIRATA ${ }^{* *}$, Kazuaki SHOBU**, Taisuke MURAYAMA ${ }^{* *}$ \\ and Jiro FUNAKI ${ }^{* *}$ \\ ${ }^{* *}$ Department of Mechanical Engineering, Doshisha University \\ 1-3 Tataramiyakodani, Kyoutanabe-shi, Kyoto 610-0321, Japan \\ E-mail: khirata@mail.doshisha.ac.jp
}

\begin{abstract}
The authors research the jet from an asymmetrical two-dimensional nozzle, especially concerning the effect of the lip length of the nozzle. Experiments are conducted at a Reynolds number of 6000 . The aspect ratio of the nozzle exit is fixed to 300 . And, the lip length is $0,2.0 h, 3.3 h$ and $5.0 h$, where $h$ denotes the height of the nozzle exit. Using a hot-wire anemometer, the authors show mean-velocity and turbulence-intensity profiles at various downstream sections, in order to reveal fundamental characteristics of the jet in both the near and far downstreams. Furthermore, in the near downstream, the authors visualise the flow by glycol smoke so as to get quantitative information using a PIV technique, and perform the dominant-frequency measurements to reveal flow-instability characteristics.
\end{abstract}

Key words: Environmental Engineering, Air Conditioning, Air Curtain, Jet, Turbulent Mixing, Two-Dimensional Flow, Nozzle

\section{Introduction}

Mixing/diffusion enhancement is one of the key technologies in various environmental aspects, and has become needed in chemical reactors, heat exchangers, burners/combustors, air conditioners and so on. Jets are known to be useful for such mixing/diffusion enhancement (see Hirata et al. (2009) ${ }^{(1)}$ and Funaki et al. (2009) ${ }^{(2)}$ ). Most of past studies concern a circular jet, namely, the jet from a nozzle with a circular cross section. On the other hand, we have been recently focusing our interest upon non-circular jets, to achieve more efficient mixing/diffusion enhancement. Among non-circular jets, a plane jet has fundamental and practical importance, as it is applicable for various fields such as (1) drying/cooling of plastic films and fabrics, (2) cleaning/draining/drying of manufacturing products, (3) flow controls inside burners and at furnace entrances, and (4) efficient and smart air conditioning devices such as air curtains and air screens.

Now, we concentrate our notice upon turbulent and free-plane jets at high Reynolds numbers $R e$ 's, considering practical importance in many industrial fields. Up to now, there have been several researches concerning the turbulent and free-plane jet (for example, see Förthmann (1936), ${ }^{(3)}$ Goertler (1942), ${ }^{(4)}$ Zijnen (1958), ${ }^{(5)}$ Mi et al. (2005a) $\left.{ }^{(6)}\right)$. However, most of them are about the jet emitted from a symmetrical nozzle, and there have been only a few researches concerning the jet emitted from an asymmetrical nozzle, such as Husain \& Hussain (1983) ${ }^{(7)}$ and Kiwata et al. (2009). ${ }^{(8)}$ Our knowledge about such asymmetrical plane jets has not been enough yet, in spite of their potentials to various applications like (1) increase/decrease of the flow entrainment or the streamwise growth of flow rate, (2) promotion/suppression of the decay of flow velocity, (3) control of the jet's direction, (4) realisation of oscillatory or pulsative jets, and (5) generation of the asymmetrical flow fields concerning time-mean velocity, turbulence and so on.

In the present study, we research the turbulent and free-plane jet from an asymmetrical

${ }^{*}$ Received 2 Dec., 2009 (No. 09-0723) [DOl: 10.1299/jee.5.183]

Copyright $\odot 2010$ by JSME 
two-dimensional nozzle, which has a lip only on one side of the nozzle. We especially focus our attention upon the effect of the lip length. Among various control methods to asymmetrise a plane jet, such as (1) asymmetrical nozzle geometry, (2) insertion of a downstream object or a nozzle-surface object and (3) addition of secondary jets, the lip-length control has advantages of both (1) simple geometry with less control parameters and (2) easier change of control-parameter values. Experiments are conducted at a Reynolds number $R e$ of 6000 . The aspect ratio $A R$ of the nozzle exit is fixed to 300. And, the lip length $l$ is $0,2.0 h, 3.3 h$ and $5.0 h$, where $h$ denotes the height of the nozzle exit. Using a hot-wire anemometer, we measure mean-velocity and turbulence-intensity profiles at various downstream sections, to reveal fundamental characteristics of the jet in both the near and far downstreams. Besides, in the near downstream, we visualise the jet by glycol smoke so as to get quantitative information using a particle-image velocimetry (hereinafter, referred to as PIV) technique, and perform the dominant-frequency measurements to reveal flow-instability characteristics.

\section{Nomenclature}

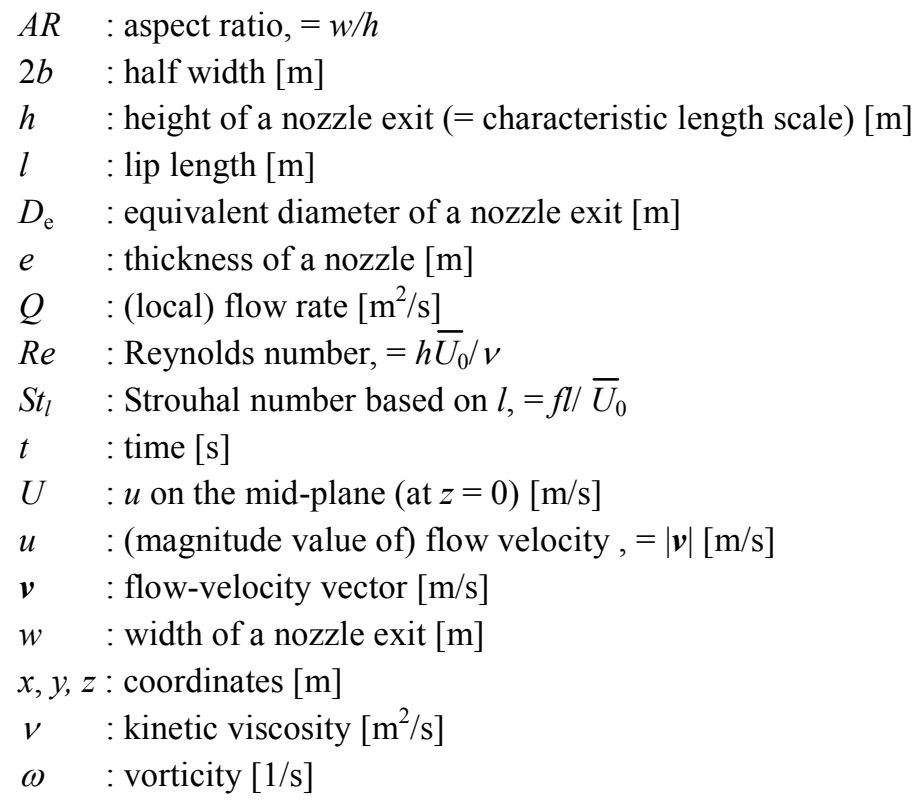

Superscript

- : time-mean

Subscripts

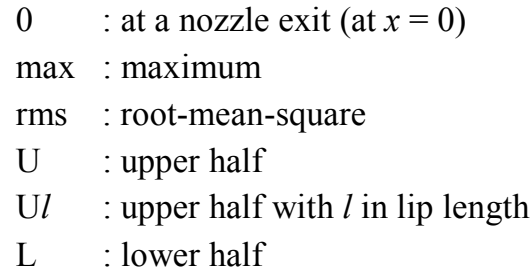

\section{Experimental Method}

\subsection{Model and coordinate system}

Figure 1 shows the present model, namely, a plane jet issued into an open-space and stationary fluid from an asymmetrical two-dimensional nozzle with a different dimension $l$ 
between both the ends of the upper and lower halves of a nozzle, which is hereinafter called as a "lip length." In the present study, the upper half of the nozzle is longer than the lower half by $0,2.0 h, 3.3 h$ and $5.0 h$, where $h\left(=1.5 \times 10^{-3} \mathrm{~m}\right)$ represents the height of the nozzle exit, which is used as a characteristic length scale.

Figure 1 also shows the present coordinate system, together with important physical parameters. The coordinate's origin $\mathrm{O}$ is at the nozzle exit on the mid-plane and on the span-centre plane. Strictly speaking, "the nozzle exit" is at the end of the lower half of the nozzle. The coordinate system is a Cartesian one with a streamwise component $x$, a cross-streamwise (span-wise) component $y$ and another cross-streamwise (transverse) component $z$.

In general, flow-velocity vector $v$ at an arbitrary location is a function of $x, y, z$ and $t$, where $t$ is time. Supposing the two-dimensionality of statistical flow field, we usually consider only the span-centre plane at $y=0$ (see Fig. 3, for reference). Then, $v$ could be the function of $x, z$ and $t$. $U$ denotes the flow velocity on the mid-plane (at $z=0$ ), then, is a function of $x$ and $t$ alone. A subscript " 0 " denotes the location of the nozzle exit. So, $U_{0}$ is a function of $t$ alone. We define the magnitude value $u$ of flow velocity as $|\boldsymbol{v}|$. Both $\bar{u}$ and $u_{\text {rms }}$ are functions of $x$ and $z$ alone, where a superscript "-" and a subscript "rms" denote time-mean and root-mean-square, respectively. The half width $2 b$ on the transverse velocity profile is defined as the width at whose boundaries $\bar{u}=\bar{u}_{\max } / 2$, where a subscript "max" denotes the maximum value of $u$ at each $x$.

Figure 2 shows the details of the nozzle-cross-section geometry. Even for $l=0$, the nozzle has not achieve a perfect symmetry; that is, the thickness $e_{\mathrm{U} l}$ at the end of the upper half of the nozzle is slightly different from the thickness $e_{\mathrm{L}}$ at the end of the lower half of the nozzle, except for $l=3.3 \mathrm{~h}$. This slight asymmetry in nozzle geometry could bring some asymmetry in the jet, as will be shown later.

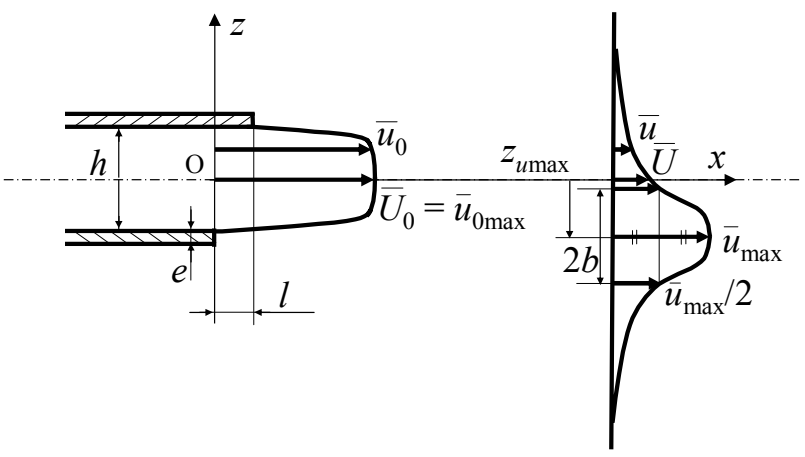

Fig. 1 Model and coordinate system.

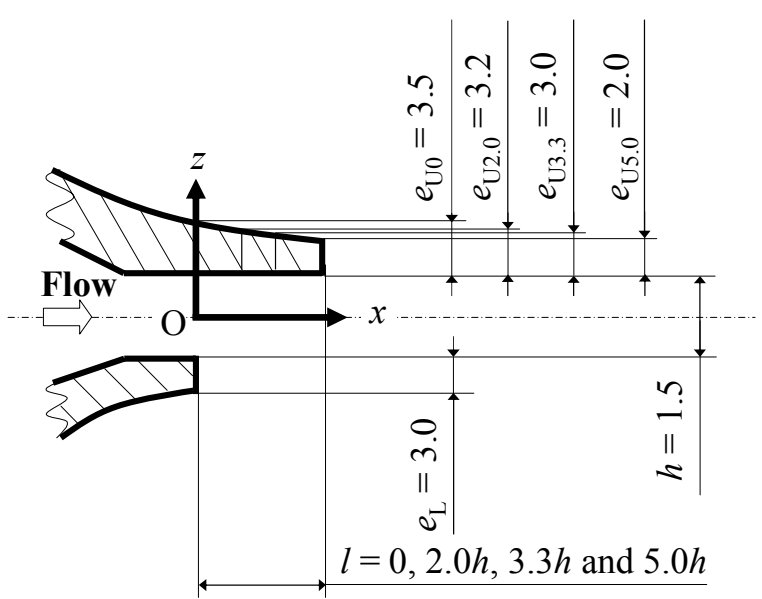

Fig. 2 Details of nozzle geometry (unit: $\mathrm{mm}$ ). 
Table 1 Experimental parameters.

\begin{tabular}{c|c}
\hline$h(\mathrm{~m})$ & $1.5 \times 10^{-3}$ \\
\hline$l(\mathrm{~m})$ & $0.0,3.0 \times 10^{-3}, 5.0 \times 10^{-3}, 7.5 \times 10^{-3}$ \\
\hline$w(\mathrm{~m})$ & $450 \times 10^{-3}$ \\
\hline$D_{\mathrm{e}}(\mathrm{m})$ & $29.3 \times 10^{-3}$ \\
\hline$U_{0}(\mathrm{~m} / \mathrm{s})$ & 60 \\
\hline$\theta(\mathrm{m})$ & $0.03 \times 10^{-3}-0.06 \times 10^{-3}$ \\
\hline$l / h$ & $0,2.0,3.3,5.0$ \\
\hline$A R(=w / h)$ & 300 \\
\hline$D{ }^{d} h$ & 19.5 \\
\hline$R e$ & 6000 \\
\hline$R e_{\theta}$ & $31-222$ \\
\hline$S t_{l}$ & $0.2-3.1$ \\
\hline$S t_{\theta}$ & $0.003-0.020$ \\
\hline Sidewalls & No \\
\hline \multicolumn{2}{|c}{}
\end{tabular}

\subsection{Experimental parameters}

As a geometric control parameter of the concerning jet, we consider a reduced lip length $l / h$. And, as a kinetic control parameter, we consider the Reynolds number Re. Its definition is as follows.

$$
R e=\bar{U}_{0} h / v,
$$

where a characteristic velocity scale $\bar{U}_{0}$ denotes the time-mean velocity at the nozzle exit on the mid-plane and on the span-centre plane.

When we consider streamwise variations of the flow, we suppose a flow rate $\bar{Q}$ a locally-integral amount of $\bar{u}$. Specifically speaking, $\bar{Q}$ is the time-mean local flow rate per unit span through a transverse cross section at a certain $x$.

Table 1 summarises the values of main experimental parameters in dimensional and non-dimensional forms. The time-mean averaging is carried out over more than $20 \mathrm{sec}$, to ensure enough accuracy and reproducibility. All the experiments are conducted for $l / h=0$, 2.0, 3.3 and 5.0 at $R e=6.0 \times 10^{3}$. At such a specified value of $R e$, the $R e$ effect might be small (see ref. ${ }^{(9)}$ ), while thorough discussion should be carried out elsewhere. We non-dimensionalise the dominant frequency $f$ of the flow-velocity fluctuation as the Strouhal number $S t_{l} \equiv f l / \bar{U}_{0} . \theta$ denotes the momentum thickness at the nozzle exit, and $D_{\mathrm{e}}$ denotes the equivalent diameter at the nozzle exit which is defined by $(4 w h / \pi)^{1 / 2}$, where $w$ is the width of the nozzle exit.

\subsection{Experimental apparatus}

In the present experimental apparatus, working fluid is air. Air is driven by a blower. And, through an enough-long straight pipe with a constant cross-section area of $1.8 \mathrm{~m}$ in length and $56 \mathrm{~mm}$ in inner diameter, air is issued out of an asymmetrical two-dimensional nozzle into stationary open space with a fully-developed velocity profile. The nozzle has such a large aspect ratio $A R(=w / h)$ as 300 . Using a hot-wire anemometer with an I-type probe whose temperature effect is compensated by a cold-wire probe, we measure $u$ at many locations downward the nozzle exit. The insertion angle of the I-type probe is determined so as to minimise the disturbance by the probe.

Using flow visualisation and a PIV technique, we observe flow structures. Specifically speaking, the tracer smoke produced by heating ethylene-glycol is introduced at the blower, and is ejected with air from the nozzle. Such smoke is lightened up by a sheet-like beam with a thickness of $1 \mathrm{~mm}$ from a double-pulse YAG laser. We record the flow visualised by 
the smoke using a CCD camera synchlonised with the laser device, and analyse it using a PIV technique on a PC.

\section{Results and Discussion}

\subsection{Two-dimensionality of flow}

At first, we confirm two-dimensionality of the flow from the present nozzle. Figure 3 shows mean-velocity profiles in the $y$ direction, namely, the span-wise direction, for four kinds of nozzles with $l / h=0,2.0,3.3$ and 5.0. Specifically speaking, the axis of ordinate denotes the maximum value in the transverse profile of $\bar{u}$ at each $y$, which is normalised by the value at $y=0$. The results for $l / h=0,2.0$ and 3.3 are measured at $x / h=33.3$, and those for $l / h=5.0$ are at $x / h=0$ (for the $z$-direction profile, see Fig. 4). Here, we especially show the results for $l / h=5.0$ at a different value of $x / h$, so as to represent that the influence of $x / h$ upon the two-dimensionality is small. As a result, we can confirm good two-dimensionality for all the tested nozzles. This will be confirmed again in Fig. 7, in comparison with other researchers. Then, from now on, we discuss only the results at $y / h=$ 0 .

\subsection{Mean-velocity profiles}

In this and following sections, we consider streamwise distributions of some quantities such as $\bar{u}$ and $u_{\text {rms }}$, standing on the measurements by a hot-wire anemometer at various values of $x / h$ and $z / h$ on the span-centre plane.

Figure 4 shows typical examples of transverse profiles of $\bar{u}$. Specifically speaking, figures (a), (b), (c) and (d) are for $l / h=0,2.0,3.3$ and 5.0, respectively.

At first, we see Fig. 4(a). At the nozzle exit (at $x / h=0$ ), a clear potential core of the jet exists at $z / h=-0.5-0.5$. Supplementarily speaking, slower flow exists in a wide range of $z / h \geq \pm 2$. Of course, we have confirmed that $\bar{u}$ asymptotes to zero at $z / h= \pm \infty$. The slower flow is considered to be related with the entrainment of ambient fluid into the jet. This will be discussed in Fig. 5. As $x / h$ increases from zero, the profile becomes gradual, that is, its peak becomes low and its foot becomes wide. As well as the profile at $x / h=0$, the profiles at $x / h \neq 0$ accompany the slower flow related with the entrainment. In addition, we can see that the profile centre monotonously shifts to the negative $z / h$ direction with increasing $x / h$. This monotonous jet bias could be owing to the incompleteness of the symmetry in nozzle geometry even for $l / h=0$, as shown in Fig. 2. Of course, the monotonous jet bias is not related with the experimental inaccuracy concerning the nozzle-axis direction (see Fig. 8).

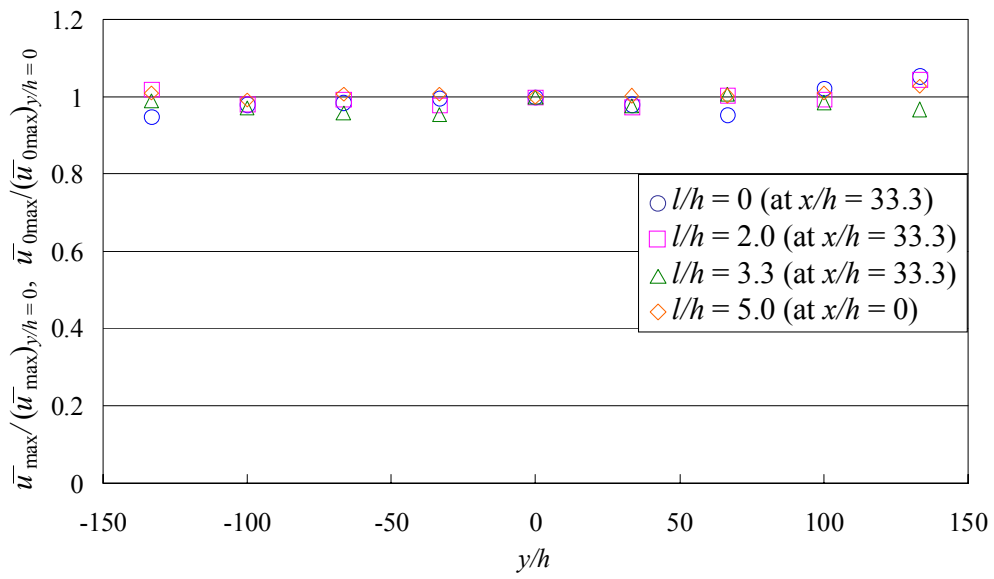

Fig. 3 Mean-velocity profiles in the $y$ direction $(l / h=0,2.0,3.3$ and 5.0, $R e=6000, A R=300)$. 
Next, we see Fig. 4(b), which shows the same features as Fig. 4(a), that is, (1) the clear potential core, (2) the slower flow related with the entrainment, (3) profile gradualness with increasing $x / h$ and (4) the monotonous jet bias. However, in contrast with Fig. 4(a), there is not any data at $z / h=0.5-2.7$ in the profile at $x / h=0$, due to the existence of the lip. Figure 4(c) is qualitatively the same as Fig. 4(b).

Finally, we see Fig. 4(d), which shows the same four features as Figs. 4(b) and 4(c), that is, (1) the clear potential core, (2) the slower flow related with the entrainment, (3) profile gradualness with increasing $x / h$ and (5) the lack of data due to the lip, except for (4) the monotonous jet bias. The jet bias shown in Fig. 4(d) seems to be more complicated than those in Figs. 4(a) - 4(c); namely, the profile centre does not monotonously shift to the negative $z / h$ direction with increasing $x / h$. This will be discussed in Fig. 8.

Now, we consider the similarity in transverse profiles of $\bar{u}$. Figure 5 shows sample results, that is, re-normalised mean-velocity profiles in the $z$ direction, for $l / h=3.3$ at several values of $x / h$. Specifically speaking, the figure is normalised using the local maximum velocity $\bar{u}_{\text {max }}$ for the ordinate, and using both a shifted coordinate $z$ ' instead of $z$ and a local half width $2 b$ instead of $h$ for the abscissa. Figure 5 also shows an empirical formula proposed by Zijnen $(1958)^{(5)}$ for a symmetrical nozzle. We can find that the present profiles agree well with Zijnen at $x / h \geq 6.7$, although the profile at $x / h=0$ is obviously

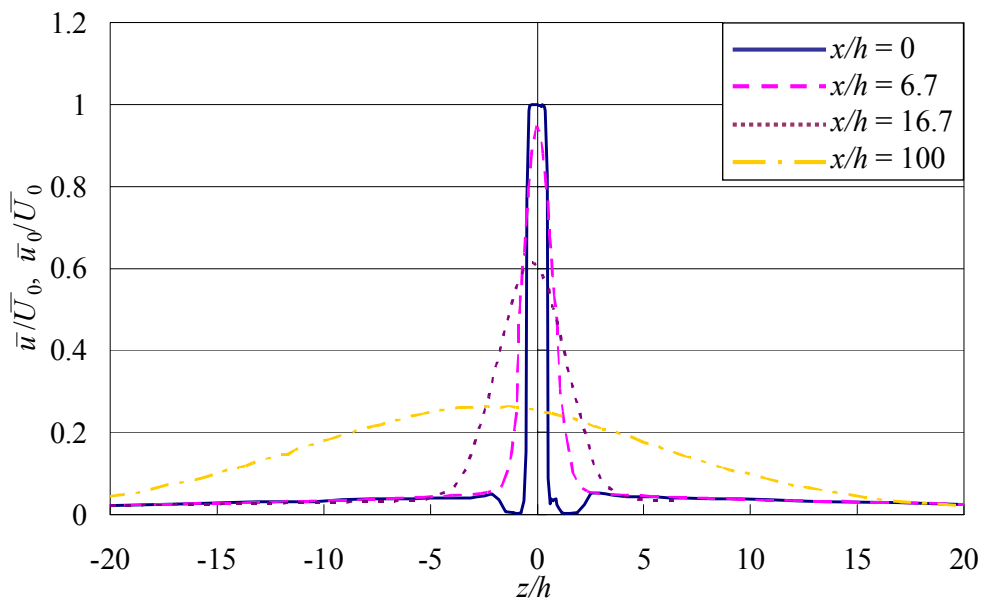

(a) $l / h=0$

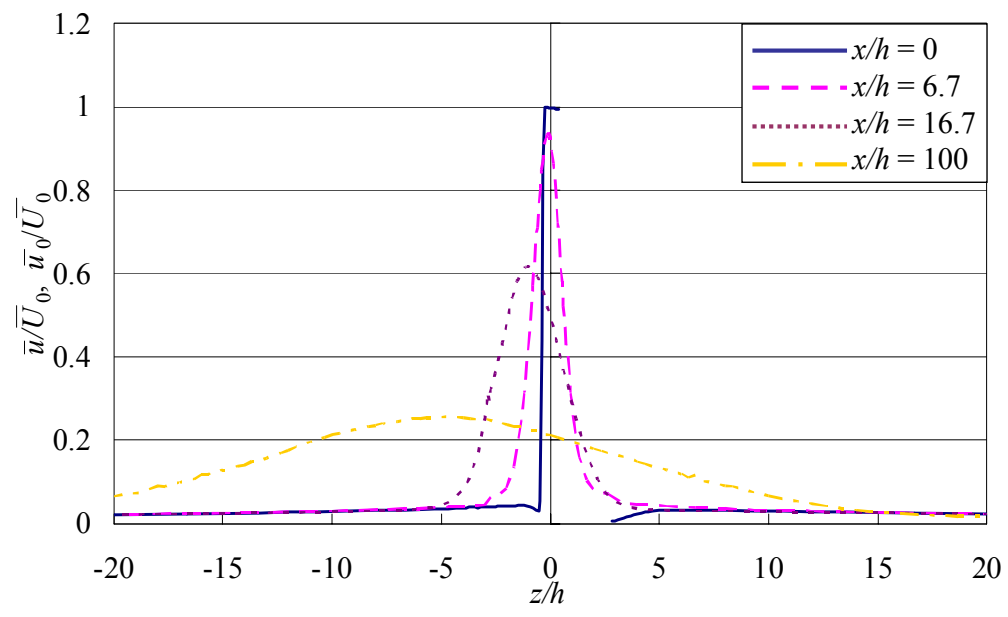

(b) $l / h=2.0$

Fig. 4 Mean-velocity profiles in the $z$ direction at $y / h=0$ $(R e=6000, A R=300)$. 
different from the others. Besides, we can find that, at $z^{\prime} / b \geq 1.5$, all the profiles at $x / h \geq 6.7$ tend to differ from Zijnen. Then, considering the difference in nozzle geometry between the present and Zijnen, we could consider that this discrepancy is owing to the entrainment of ambient fluid into the jet. Of course, the above features are shown in the other nozzles with $l / h=0,2.0$ and 5.0 .

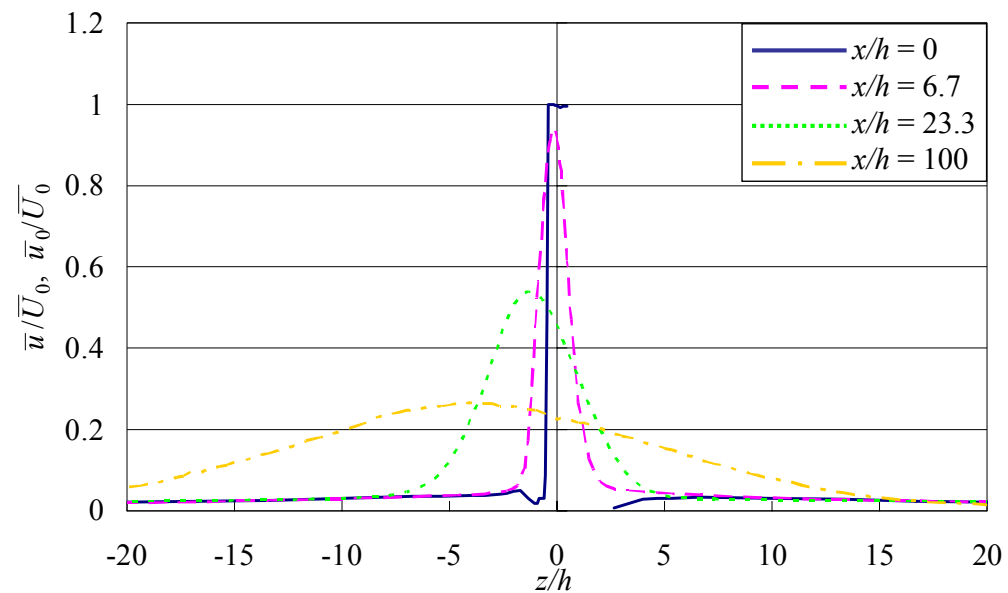

(c) $l / h=3.3$

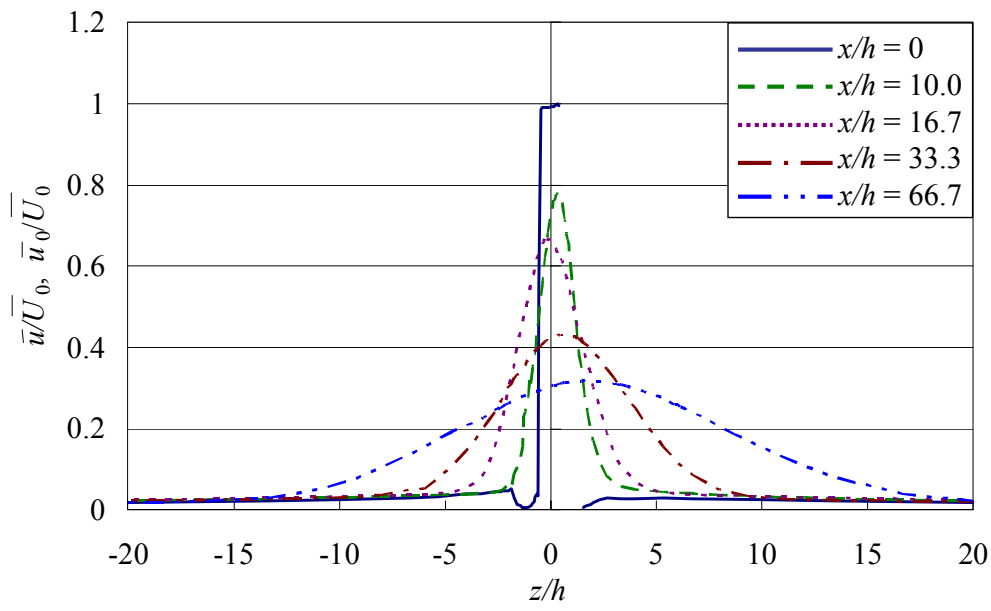

(d) $l / h=5.0$

Fig. 4 Continued

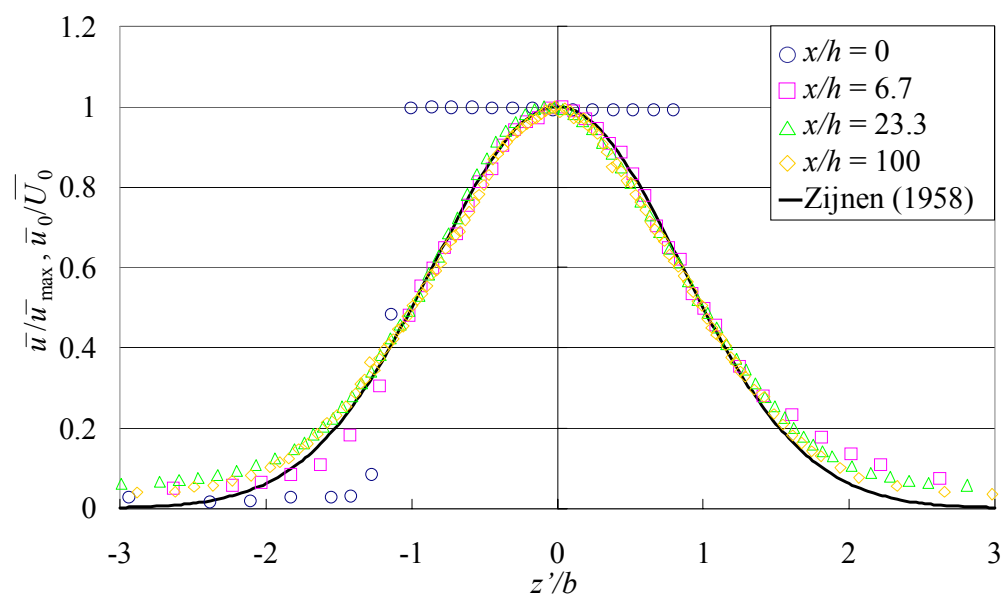

Fig. 5 Re-normalised mean-velocity profiles in the $z$ direction at $y / h=0$, $(l / h=3.3, R e=6000, A R=300)$. 


\subsection{Streamwise decay of maximum-mean-velocity}

Figure 6 shows the streamwise distributions of the maximum-mean-velocity $\bar{u}_{\max }$, for four values of $l / h$, in order to observe the streamwise variation of the jet. Figures 6 (a) and 6(b) are an overall view in a wide range of $x / h=0-120$ and a close-up view near the nozzle exit in a narrow range of $x / h=0-23.3$, respectively. Figure 6(a) shows the theory by Tollmien $(1945)^{(9)}$ for two-dimensional free jet and the experiments by Mi et al. $(2005 \mathrm{a})^{(6)}$ for symmetrical nozzles, for reference. And, Fig. 6(b) shows the Tollmien's theory and the experiments by Kiwata et al. $(2009)^{(8)}$ for asymmetrical nozzles, for reference. Table 2 summarises such other researchers' experimental parameters.

At first, we consider $\bar{u}_{\text {max }}$ in the far downstream in Fig. 6(a). All the results almost collapse on the Tollmien's theory, being independent of the values of $l / h$. This is consistent with the results by Mi et al., which approach to the Tollmien's theory with increasing $A R$.

Next, we consider $\bar{u}_{\text {max }}$ in the near downstream in Fig. 6(b). According to Rajaratnam $(1976),{ }^{(9)}$ the potential core of a two-dimensional jet exists at $x / h \leq 6$. All the present results are consistent with this, being independent of the values of $l / h$. Because, $\bar{u}_{\max } / \bar{U}_{0}$ is approximately equal to unity at $x / h \leq 6$. Supplementarily speaking, the results by Kiwata et al. ${ }^{(8)}$ do not always collapse on the Tollmien's theory. As this reason, we might consider low values of $A R$ of their nozzle exit and a large dimension of their nozzle-end thickness (also, see Table 2).

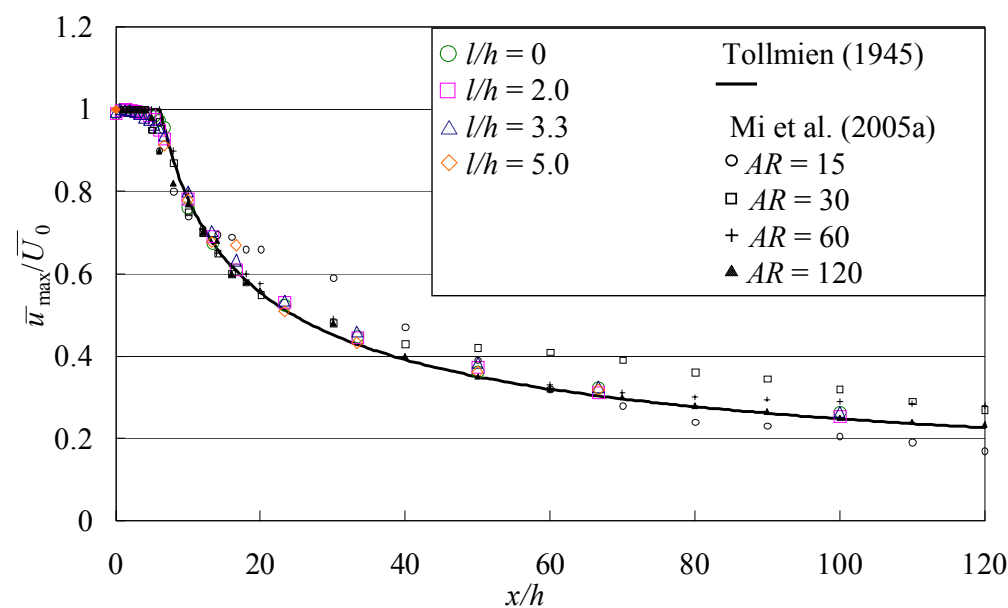

(a) Overall view $(x / h=0-120)$

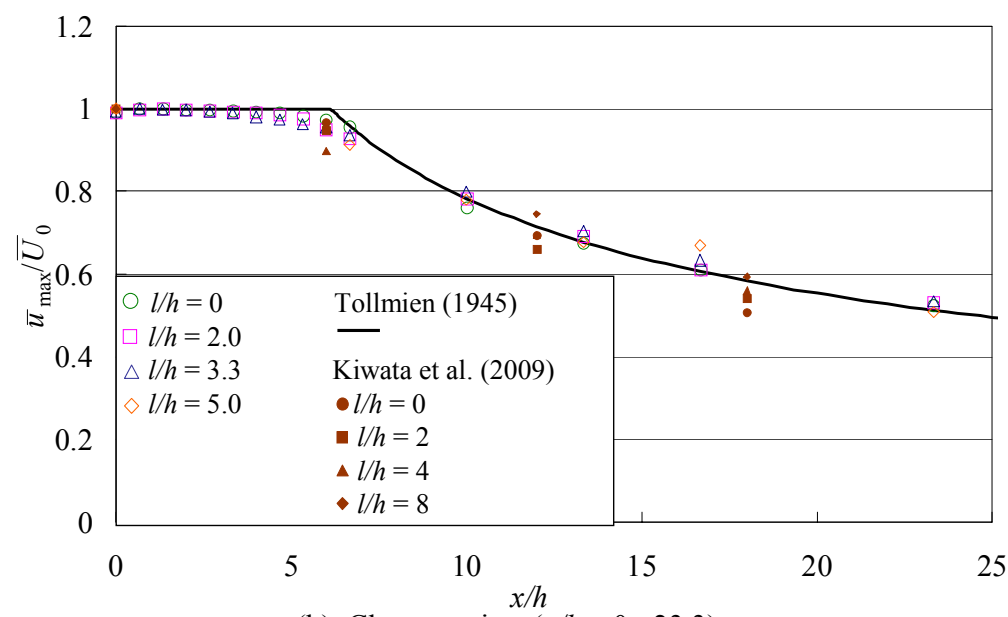

(b) Close-up view $(x / h=0-23.3)$

Fig. 6 Streamwise distributions of the maximum-mean-velocity at $y / h=0$ $(l / h=0,2.0,3.3$ and 5.0, $R e=6000, A R=300)$. 
Table 2 Other reserchers' experimental parameters. Corresponding symbols are in Figs. 6 and 7.

\begin{tabular}{|c|c|c|c|c|c|c|}
\hline Symbol & Reserchers & $R e$ & $l / h$ & $A R$ & $D_{\mathrm{e}} / h$ & Sidewalls \\
\hline$\circ$ & Husain \& Hussain (1983) & $10,700-42,700$ & unknown & 44 & 7.5 & Yes \\
\hline---- & Quinn (1992) & 208,000 & 0 & 10 & 3.6 & No \\
\hline [............ & Quinn (1992) & 208,000 & 0 & 20 & 5.0 & No \\
\hline & Deo (2005) & 18,000 & 0 & 72 & unknown & Yes \\
\hline 0 & Mi et al. (2005a) & 10,000 & 0 & 15 & 4.4 & No \\
\hline ㅁ & Mi et al. (2005a) & 10,000 & 0 & 30 & 6.2 & No \\
\hline+ & Mi et al. (2005a) & 10,000 & 0 & 60 & 8.7 & No \\
\hline$\Delta$ & Mi et al. (2005a) & 10,000 & 0 & 120 & 12.4 & No \\
\hline$\bullet$ & Kiwata et al. (2009) & 4,500 & 0.0 & 18 & 4.8 & Yes \\
\hline 口 & Kiwata et al. (2009) & 4,500 & 2.0 & 18 & 4.8 & Yes \\
\hline$\Delta$ & Kiwata et al. (2009) & 4,500 & 4.0 & 18 & 4.8 & Yes \\
\hline$\diamond$ & Kiwata et al. (2009) & 4,500 & 8.0 & 18 & 4.8 & Yes \\
\hline
\end{tabular}

Finally, in Fig. 7, we show the streamwise distributions of $\bar{u}_{\text {max }}$ with the abscissa of $x / D_{\mathrm{e}}$ instead of $x / h$. Both the abscissa and the ordinate have logarithmic scales. This figure also shows many other researchers' results, whose parameters are summarised in Table 2, again.

According to Mi et al. (2005a), ${ }^{(6)}$ we see (1) the potential core zone, (2) quasi-plane-jet zone, (3) the transition zone and (4) quasi-axisymmetric-jet zone in sequence, as $x / D_{\mathrm{e}}$ increases. And, the larger $A R$ is, the smaller the value of $x / D_{\mathrm{e}}$ where the quasi-plane-jet zone appears. Besides, the larger $A R$ is, the wider the range of $x / D_{\mathrm{e}}$ for the quasi-plane-jet zone is. In the quasi-plane-jet zone and the quasi-axisymmetric-jet zone, $\bar{u}_{\max } / \bar{U}_{0}$ is in proportion to $x^{-1 / 2}$ and $x^{-1}$, respectively.

All the present data collapse on a common straight line which is in proportion to $x^{-1 / 2}$. This suggests good two-dimensionality of the jet. In addition, we can confirm a consistency with Mi et al.; namely, the present value of $\bar{u}_{\max } / \bar{U}_{0}$ is always smaller than Mi et al. at each $x / D_{\mathrm{e}}$. This is reasonable, if we remind such a high $A R$ as 300 , which is larger than Mi et al., being independent of the values of $l / h$. Complementarily speaking, it seems difficult to compare the above results with Kiwata et al. (2009), due to their different velocity profile caused by much different nozzle geometry with a far-upstream contraction.

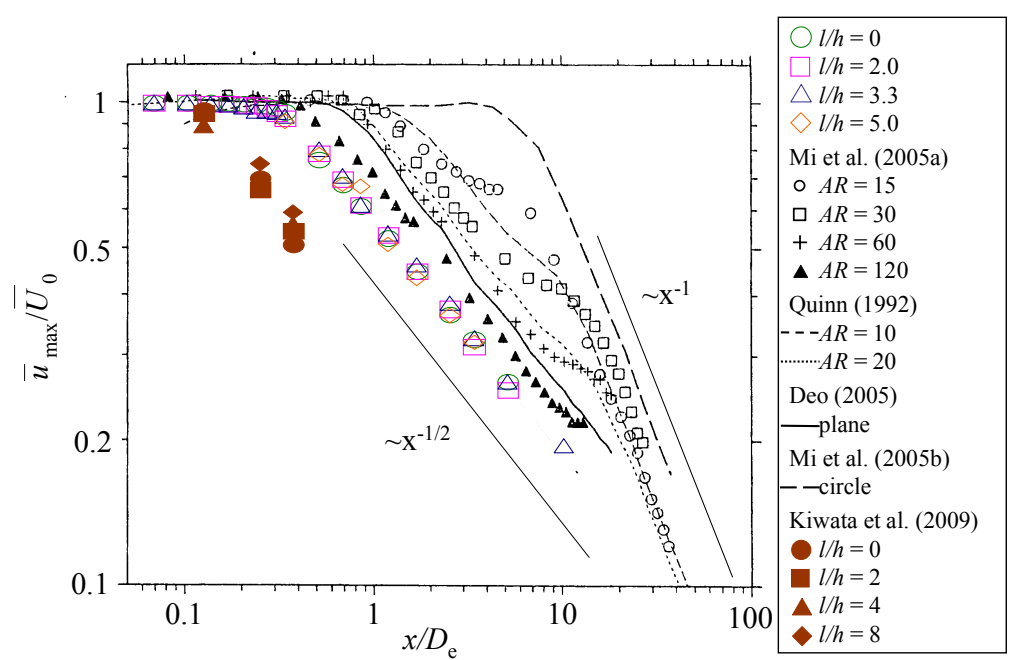

Fig. 7 Streamwise distributions of the maximum-mean-velocity at $y / h=0$, with logarithmic scales $(l / h=0,2.0,3.3$ and 5.0, $R e=6000, A R=300)$. The abscissa is normalised by an equivalent diameter $D_{\mathrm{e}}$. 


\subsection{Streamwise variation of jet bias}

Figure 8 shows the streamwise distributions of a jet bias $z_{u \max }$, namely, a local mean-velocity-profile centre, for four values of $l / h$. We define $z_{u \max }$ as the value of $z$ where the transverse mean-velocity profile attains the maximum $\bar{u}_{\max }$ at each $x$, as shown in Fig. 1. Figures $8(a)$ and $8(b)$ are an overall view in a wide range of $x / h=0-100$ and a close-up view near the nozzle exit in a narrow range of $x / h=0-16.7$, respectively. Error bars in these figures show the boundaries of the region where $\bar{u} \geq 0.95 \bar{U}_{0}$. This region could be approximately regarded as the potential core.

At first, we see Fig. 8(a). When we consider the far downstream at $x / h \geq 10$, we can clearly confirm the streamwise growth of the jet bias suggested in Fig. 4. Concretely speaking, the jet bias for $l / h=0$ is negative and is almost in proportion to $x / h$. Namely, the jet direction is not horizontal, but somewhat downward. This is considered to be related with the incompleteness of the symmetry in nozzle geometry, even for $l / h=0$. In addition, the jet axis is almost linear.

As well, the jet biases for $l / h=2.0$ and 3.3 show the above two features such as (1) the downward deflection and (2) the spatial linearity of the jet axis. However, from a quantitative viewpoint, we can find an effect of $l / h$ upon the jet bias, if we compare the results for $l / h=0,2.0$ and 3.3. Namely, by the $l / h$ effect, the large $l / h$ becomes, the more downward the jet deflection is.

In contrast with the jet biases for $l / h=0,2.0$ and 3.3, the jet bias for $l / h=5.0$ indicates two different features as follows. (1) The jet deflection is upward. And, (2) the jet axis remarkably meanders, especially at $x / h=10-30$. We should note that the jet-axis meanders can be observed for other values of $l / h$. But, they are much weaker than that for $l / h=5.0$. We might suppose the relation of the jet-axis meander to the break of flow two-dimensionality, which is expected to be revealed in future.

Next, we see Fig. 8(b) so as to consider the near downstream at $x / h<10$, where Fig. 8 (a) is not appropriate owing to condensed results at $x / h<10$. As well as Fig. 6 , we can again confirm that the present results coincide with Rajaratnam $(1976),{ }^{(9)}$ as the potential core of a two-dimensional jet exists at $x / h \leq 6$ for all the tested $l / h=0,2.0$ and 3.3.

Incidentally speaking, concerning the shape of the potential core, it seems difficult to find out any clear $l / h$ effects. Especially for $l / h=0$, the upper and lower outer boundaries of the potential core are fairly symmetrical concerning the horizontal axis $Z_{\text {umax }} / h=0$. This suggests such a fact that the nozzle axis is accurately installed parallel to the horizontal axis, while the jet bias exists in the downstream even for $l / h=0$.

\subsection{Streamwise growth of half width}

Figure 9 shows the streamwise distributions of the half width $2 b$, for four values of $l / h$. Both the axes are normalised by $h$. In the figure, an empirical formula is given for each $l / h$. For reference, the figure also shows the theory by Rajaratnam $(1976)^{(9)}$ such as

$$
b=0.10 x \text {. }
$$

At $x / h \leq 6,2 b / h$ is almost unity, being independent of $l / h$. This is consistent with the existence of the potential core. At $x / h>6,2 b / h$ increases linearly with increasing $x / h$. Moreover, we can see that the results for $l / h=0,2.0$ and 3.3 collapse on the theory by Rajaratnam for a two-dimensional free jet. On the other hand, the result for $l / h=5.0$ shows a clear $l / h$ effect, by which $2 b / h$ tends to have a larger value than those for $l / h=0,2.0$ and 3.3 and than the Rajaratnam's theory.

\subsection{Streamwise growth of flow rate}

Figure 10 shows the streamwise distribution of the local time-mean flow rate $\bar{Q}$ per unit span, for four values of $l / h$. The axis of ordinate is normalised by $\bar{Q}_{0}$, which denotes the flow rate from the nozzle exit. For reference, the figure also shows the theory by 
Albertson et al. (1950) ${ }^{(9)}$ for a two-dimensional free jet such as

$$
\frac{q}{Q}=0.44 \sqrt{\frac{2 x}{h}},
$$

and experiments by Kiwata et al. (2009) ${ }^{(8)}$ for asymmetrical nozzles.

For all $l / h$ 's, $\bar{Q} / \bar{Q}_{0}$ tends to increase monotonously with increasing $x / h$. At first, we see the results for $l / h=0$, which almost agree with Albertson et al. By this, we can confirm the accuracy of the present experiments. Next, we see the results for $l / h=2.0,3.3$ and 5.0, where $\bar{Q} / \bar{Q}_{0}$ is always larger than Albertson et al. This suggests that the flow entrainment can be enhanced by the lip. In detail, we can find a complicated $l / h$ effect upon the value of $\bar{Q} / \bar{Q}_{0}$ : that is, $\bar{Q} / \bar{Q}_{0}$ for $l / h=2.0$ is the largest among those for other $l / h$ ' $\mathrm{s}$ at $x / h<10$; $\bar{Q} / \bar{Q}_{0}$ for $l / h=3.3$ is the largest at $x / h=10-15 ; \bar{Q} / \bar{Q}_{0}$ for $l / h=5.0$ is the largest at $x / h$ $>15$. This corresponds to the fact that the increasing manner of $\bar{Q} / \bar{Q}_{0}$ with increasing $x / h$ is affected by the value of $l / h$, although $\bar{Q} / \bar{Q}_{0}$ monotonously increases with increasing $x / h$ for any $l / h$.

To summarise the above, it seems difficult to directly connect the maximum-mean velocity, the jet bias, the half width and the local flow rate, one another.

\subsection{Turbulence-intensity profile}

Figure 11 shows a typical example of transverse profiles of $u_{\mathrm{rms}}$. Specifically speaking, this figure is for $l / h=3.3$ at several values of $x / h$. At the nozzle exit (at $x / h=0$ ), a sharp

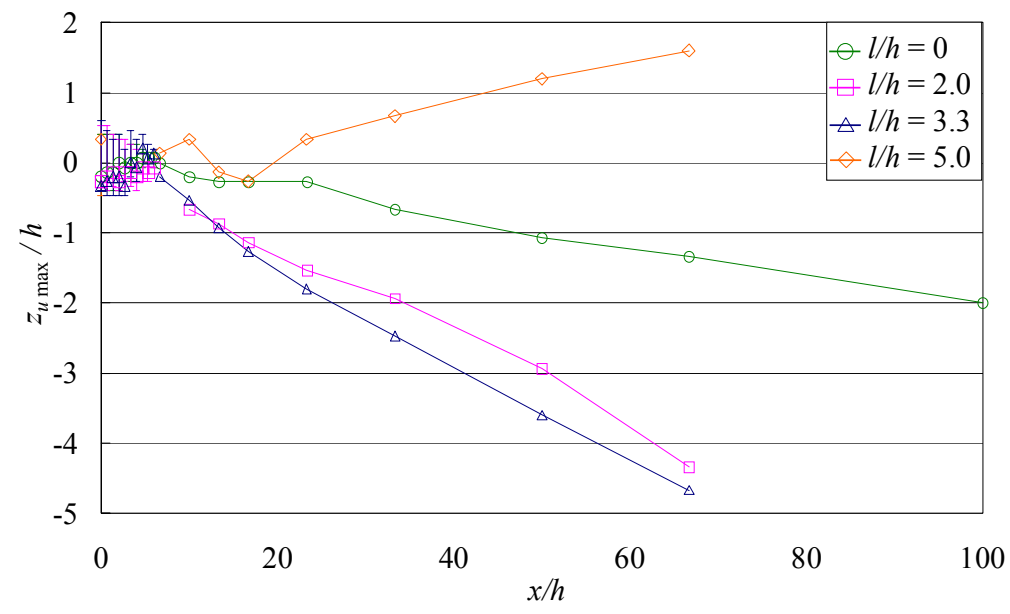

(a) Overall view $(x / h=0-100)$

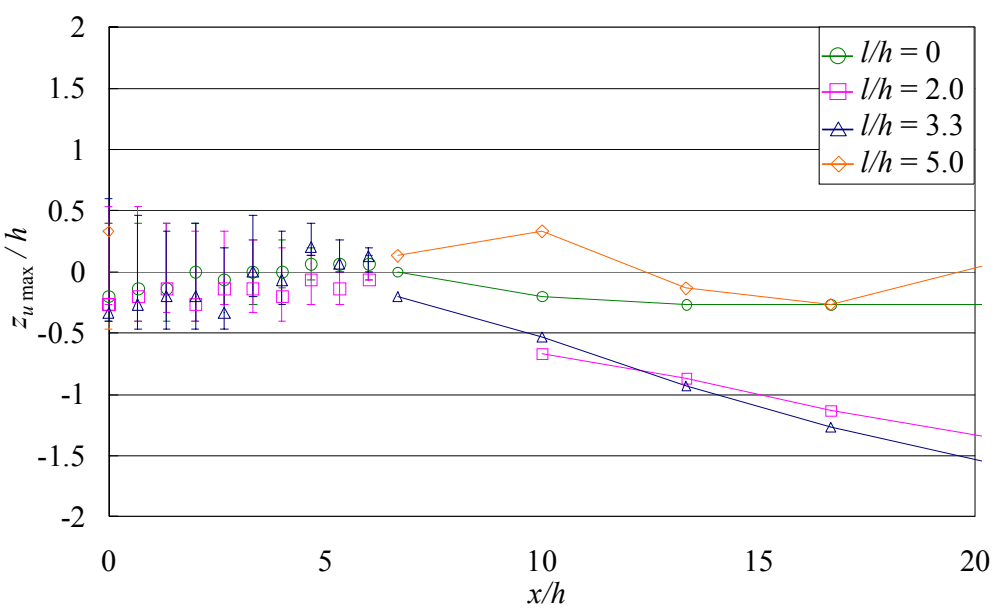

(b) Close-up view $(x / h=0-16.7)$

Fig. 8 Streamwise distributions of a jet bias $z_{\text {umax }}$ at $y / h=0$ $(l / h=0,2.0,3.3$ and 5.0, $R e=6000, A R=300)$. 
peak exists at $z / h=-0.5$, which corresponds to a shear layer on the potential-core boundary. At $x / h=3.3$, another sharp peak exists at $z / h=0.5$, where the peak value of $u_{\text {rms }} / \bar{u}_{\text {max }}$ is larger than that at $z / h=-0.5$. As $x / h$ increases from 3.3 , both the two peak values of $u_{\mathrm{rms}} / \bar{u}_{\max }$ and both the widths of their feet increase.

In addition, we can confirm both (1) the profile asymmetry and (2) the profile-centre bias. The former is represented by the difference between the two peak values of $u_{\text {rms }} / \bar{u}_{\max }$, and is quenched at such a large $x / h$ as 100 . Till the quench, the superiority in the value of $u_{\text {rms }} / \bar{u}_{\text {max }}$ usually switches between the two peaks (to be discussed in Fig. 12). The latter intensifies with increasing $x / h$, as well as the profile of $\bar{u} / \bar{U}_{0}$. The above features are commonly seen for other values of $l / h$.

\subsection{Streamwise decay of turbulence-intensity asymmetry}

Figure 12 shows the streamwise distributions of the difference between the two peak values of the $\left(u_{\mathrm{rms}}\right)_{\mathrm{Umax}}$ and $\left(u_{\mathrm{rms}}\right)_{\mathrm{Lmax}}$ for four values of $l / h$, to observe the streamwise variation of the profile asymmetry. The subscripts "Umax" and "Lmax" denote the turbulence-intensity peaks at larger $z / h$ and smaller $z / h$, respectively. Figures 12 (a) and 12(b) are an overall view in a wide range of $x / h=0-100$ and a close-up view near the nozzle exit in a narrow range of $x / h=0-16.7$, respectively.

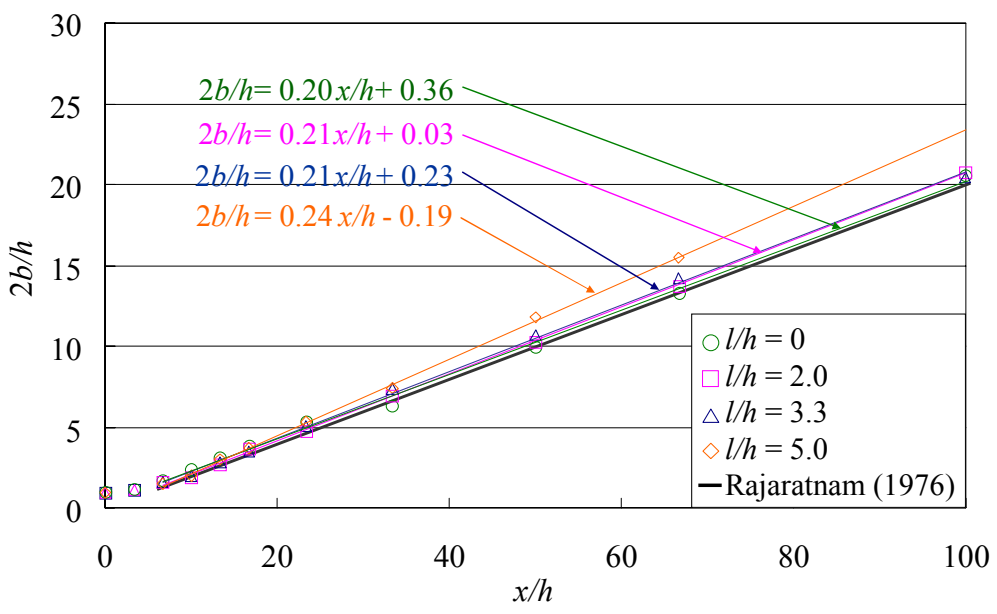

Fig. 9 Streamwise distributions of a half width $2 b$ at $y / h=0$ $(l / h=0,2.0,3.3$ and 5.0, $R e=6000, A R=300)$.

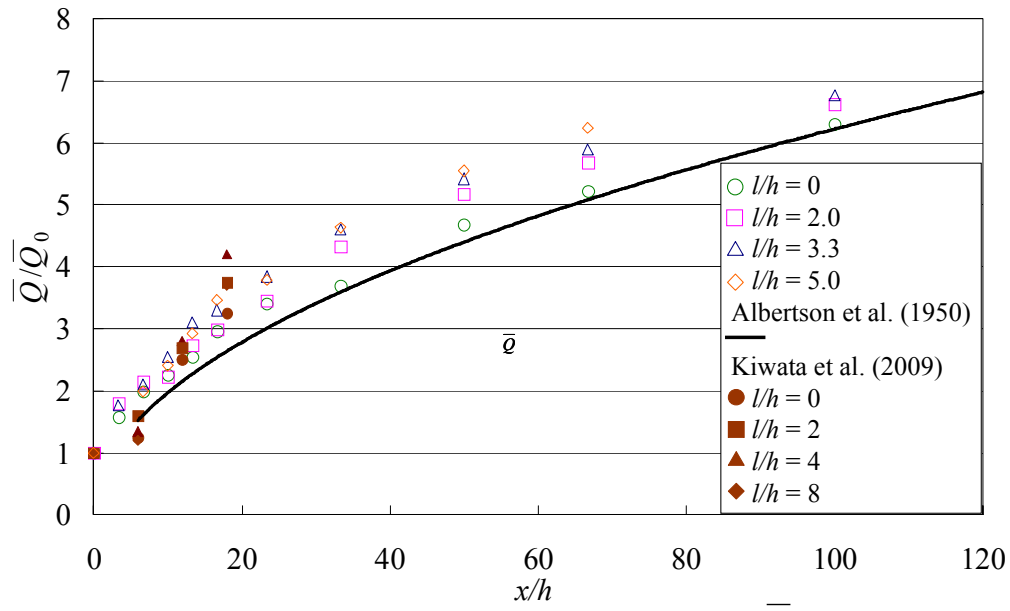

Fig. 10 Streamwise distributions of a local flow rate $\bar{Q}$ at $y / h=0$ $(l / h=0,2.0,3.3$ and 5.0, $R e=6000, A R=300)$. 
At first, we see Fig. 12(a). The difference is almost zero in the far downstream at $x / h>$ 20 , for all the values of $l / h$. However, at $x / h \leq 20$, the difference varies with $x / h$ for each $l / h$ in a complicated manner, which is considered to be related with a complicated shear layers near the potential-core end.

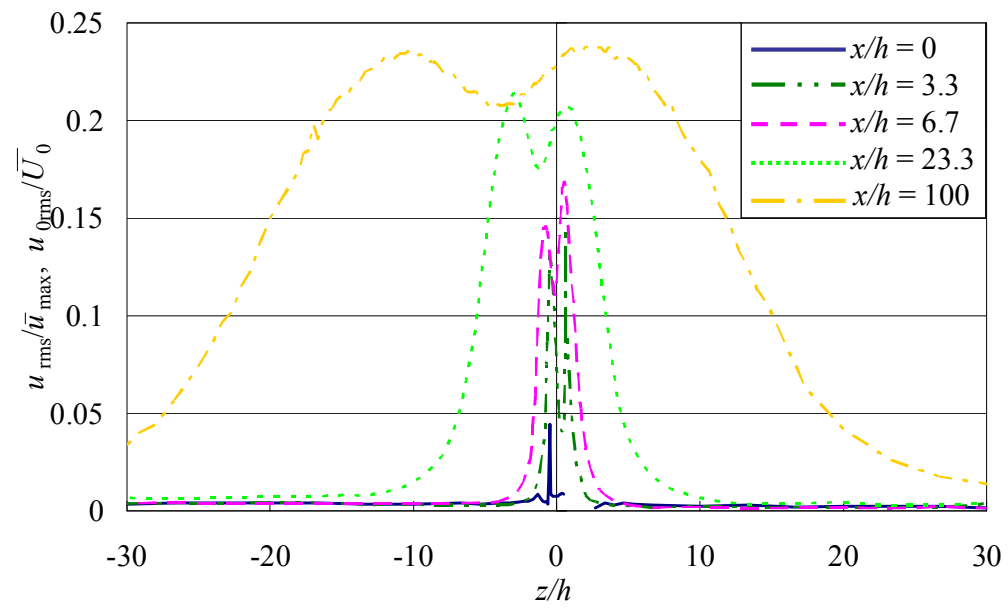

Fig. 11 Turbulence-intensity profiles in the $z$ direction at $y / h=0$ $(l / h=3.3, R e=6000, A R=300)$.

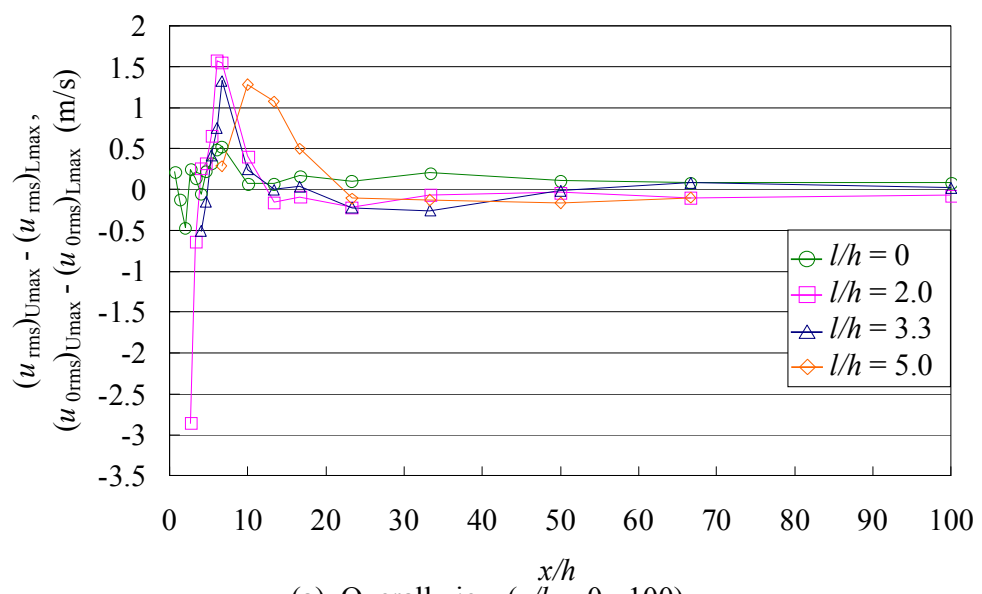

(a) Overall view $(x / h=0-100)$

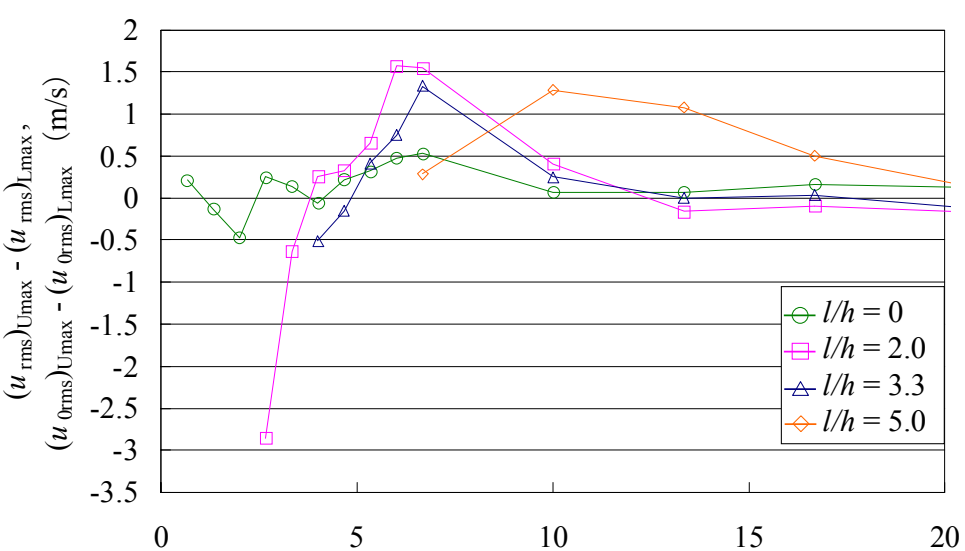

(b) Close-up view $(x / h=0-16.7)$

Fig. 12 Streamwise distributions of the difference between two turbulence-intensity peak values at $y / h=0$ $(l / h=0,2.0,3.3$ and 5.0, $R e=6000, A R=300)$. 
Then, we see Fig. 12(b) so as to consider the near downstream at $x / h \leq 20$. At $x / h \leq 6$, where the potential core exists, the difference tends to increase from the negative to the positive, as $x / h$ increases. This can be confirmed for $l / h=0,2.0$ and 3.3, while the tendency is not so clear for $l / h=0$. Then, at the end of the potential core, the upper peak of the turbulence-intensity profile is superior to the lower peak. At $x / h>6$ where the potential core disappears enhancing the interaction between the upper and lower shear layers, the difference decreases monotonously approaching to zero with increasing $x / h$ for $l / h=0,2.0$, 3.3 , except for $l / h=5.0$. This suggest a certain singularity of the flow for $l / h=5.0$ from the other $l / h$ 's. Namely, for $l / h=5.0$, the difference does not monotonously decrease, but attains the maximum at $x / h=10$. Such a singularity in the turbulence-intensity-peak difference for $l / h=5.0$ well corresponds to those in the jet bias (in Fig. 8) and the half width (in Fig. 9), although we can not see any singularities for $l / h=5.0$ in the mean-velocity-profile similarity, the maximum-mean velocity and the local flow rate. We might expect that future instantaneous flow analyses will show an insight concerning such complicated relations among statistical quantities.

\subsection{Flow visualisation}

In the near downstream, the concerning flow involves obvious large-scale flow structures. Figure 13 shows a pair of sample photographs for flow visualisation. Specifically speaking, the figure is for $l / h=3.3$ at two different instances $t_{1}$ (Fig. 13(a)) and $t_{2}$ (Fig. 13(b)).

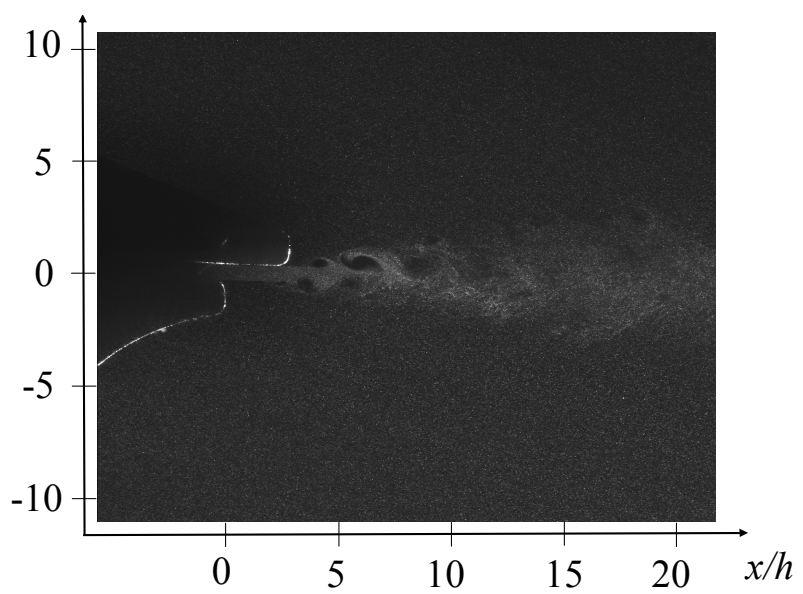

(a) $t=t_{1}$

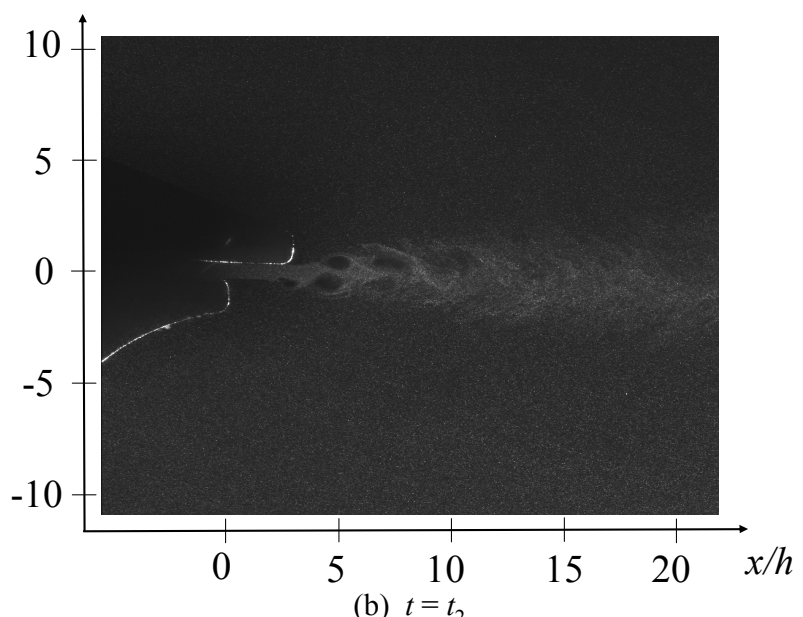

Fig. 13 Visualised flow at $y / h=0$ (side view) $(l / h=3.3, R e=6000, A R=300)$. 
We can see a street of vortical structures, which is in neither an alternate arrangement nor a symmetrical arrangement. Positions of the vortical structures at $t=t_{1}$ differ from those at $t=t_{2}$, because of the downward convection of the vortical structures.

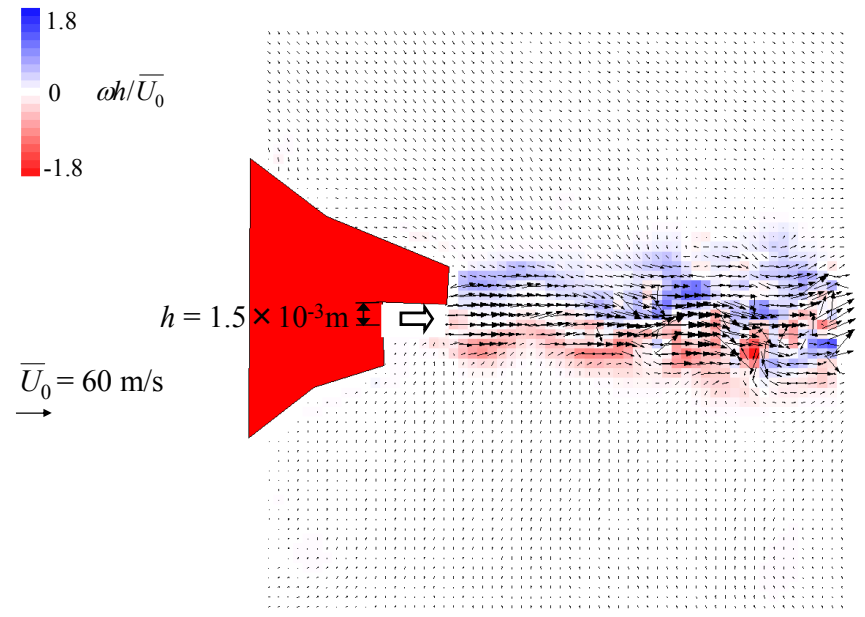

(a) $t=t_{1}$

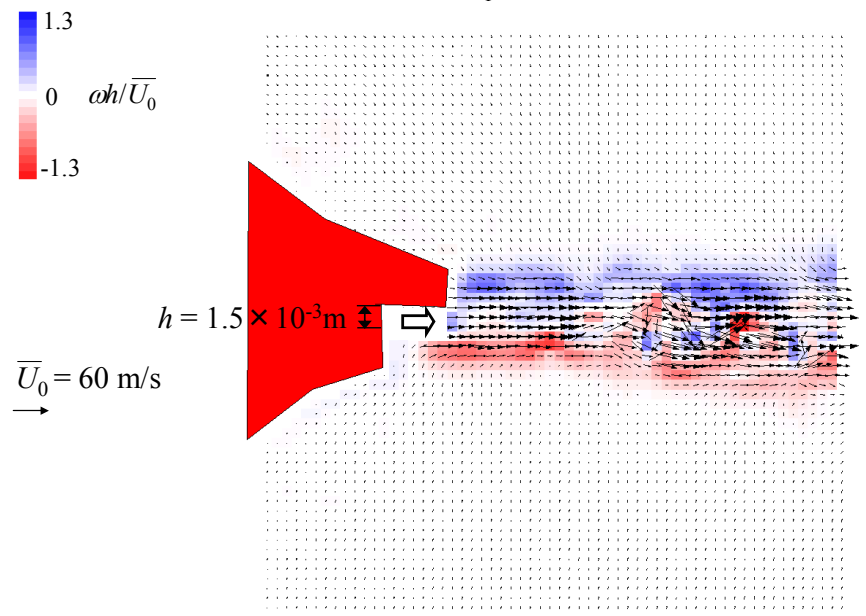

(b) $t=t_{2}$

Fig. 14 Velocity vectors and vorticity denseness at $y / h=0$ (side view) $(l / h=3.3, R e=6000, A R=300)$.

$t_{1}$ and $t_{2}$ correspond to those in Fig. 13, respectively.

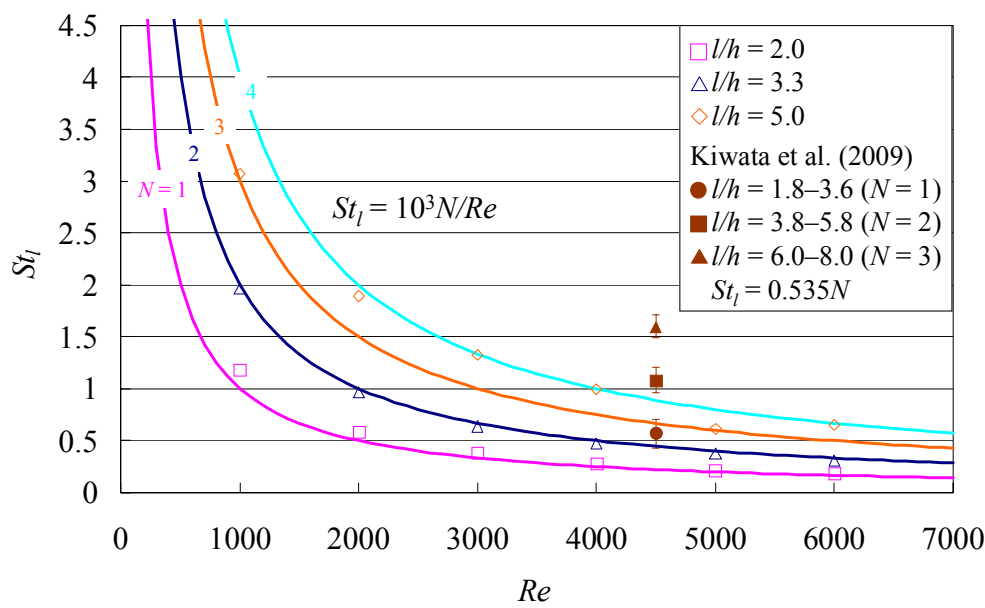

Fig. 15 Strouhal number $S t_{l}$ based on lip length versus Reynolds number $R e$ $(x / h=1.3-4.7, z / h=-0.4--2.7, y / h=0, l / h=2.0,3.3$ and 5.0, $A R=300)$. 
Figure 14 shows the spatial distributions of velocity vectors and vorticity denseness, which are obtained by the PIV analyses. This figure corresponds to Fig. 13. We can confirm both (1) the jet undulation related with the vortical-structure street, and (2) the entrainment of ambient fluid into the jet, near whose boundaries most of velocity vectors are inflowing.

\subsection{Strouhal number}

Just in the near downstream, the jet fluctuates with a dominant frequency due to the vortical-structure street visualised in Figs. 13 and 14. According to Kiwata et al., ${ }^{(8)}$ the dominant frequency is characterised by the edge-tone instability. ${ }^{(9),(10)}$ Then, Kiwata et al. have proposed such an empirical formula as

$$
S t_{l}=0.535 N
$$

where $S t_{l}$ and $N$ denote the Strouhal number based on the lip length $l$ instead of $h$ and integer, respectively. This represents that $S t_{l}$ varies discretely corresponding to the value of $N$.

Figure 15 shows $S t_{l}$ plotted against $R e$, for $l / h=2.0,3.0$ and 5.0. For $l / h=0$ we can not clearly detect any dominant frequencies. The present detected area is at $x / h=1.3-4.7$ and at $z / h=-0.4--2.7$. To conclude, all the data collapse on such a proposed empirical formula as

$$
S t_{l}=10^{3} \mathrm{~N} / \mathrm{Re},
$$

where the $R e$ effect is also taken into consideration. Therefore, we can see that all the dominant frequencies in the near downstream could be characterised only by the edge-tone instability. In this context, we can not recognise any singularities for $l / h=5.0$ in the periodic phenomenon in the near downstream. Supplementarily speaking, experimental values of $S t_{l}$ by Kiwata et al. are much larger than the present ones, due to the difference in nozzle geometry (also see Fig. 7).

\section{Conclusions}

We have researched the turbulent and free-plane jet from an asymmetrical two-dimensional nozzle. We focus our attention on the effects of the lip length upon fundamental characteristics of the jet in both the near and far downstreams. As a result, we obtain the following.

1. We have successfully realised such an sufficiently-two-dimensional jet as the quasi-plain jet by Mi et al., for the reduced lip length $l / h=0-5.0$.

2. All the normalised time-mean-velocity profiles at $x / h \geq 6.7$ show good similarity with that by Zijnen for a two-dimensional symmetrical nozzle. However, far from the profile center the similarity becomes poor, due to the entrainment of ambient fluid into the jet.

3. All the streamwise decays of the maximum value $\bar{u}$ max in the transverse time-mean-velocity profile coincide with the Tollmien's theory in both the near and far downstreams, being independent of $l / h$.

4. The jet biases for $l / h=0,2.0$ and 3.3 show two common features such as (1) a downward deflection and (2) a spatial linearity of the jet axis. There exists such a $l / h$ effect as the jet bias increases with increasing $l / h$. The jet bias for $l / h=5.0$ is exceptional, such as features as (1) an upward deflection and (2) a meander of the jet axis.

5. The streamwise growth of the half width agree well with the Rajaratnam's theory, for $l / h$ $=2.0$ and 3.0.The half width for $l / h=5.0$ shows such a singularity as larger half width, again.

6. The time-mean-local-flow rates for $l / h=2.0,3.3$ and 5.0 are larger than Albertson et al., which is equal to that for $l / h=0$. The effect of $l / h$ on the time-mean flow rate is complicated.

7. The streamwise decay of the turbulence-intensity asymmetry shows a singularity only for 
$l / h=5.0$, seems to which correspond to the singularities for $l / h=5.0$ in the jet bias and the half width. However, we can not see any singularities for $l / h=5.0$ in the mean-velocity profile similarity, the maximum-mean-velocity and the local flow rate.

8. In the near downstream, the jet fluctuates with a dominant frequency due to a visualised vortical-structure street. The dominant frequency is always characterised by the edge-tone instability, even for $l / h=5.0$. And, we have proposed an empirical formula, taking the $R e$ effect into consideration.

The present study has revealed the potential for various smart jet's controls. However, it is difficult to directly connect the maximum-mean velocity, the jet bias, the half width, the local flow rate and the turbulence intensity asymmetry, one another. We might expect that future fine and wide-ranged studies will extend the potential of asymmetrical two-dimensional nozzles.

\section{Acknowledgements}

We appreciate much useful discussions at "International Workshop on Environment \& Engineering 2009," where this paper was presented.

\section{References}

(1) K. Hirata, J. Funaki, T. Kubo, Y. Hatanaka, M. Matsushita and K. Shobu, "An experimental study of amplitude and frequency effects upon a pulsating jet," J. Fluid Science \& Technology, Vol. 4, No. 3, pp. 578-588 (2009).

(2) J. Funaki, D. Kobayashi, K. Shobu and K. Hirata, "Consecutive PIV analyses on a finite-amplitude pulsating jet," J. Fluid Science \& Technology, Vol. 4, No. 3, pp. 673-686 (2009).

(3) E. Förthmann, “Turbulent jet expansion,” NACA. TM. 789 (1936).

(4) H. Goertler, "Berechnung von aufgaben der freien turbulenz auf grund eines neuen naherungsansatzes," ZAMM, Vol. 22, No. 5, pp. 244-254 (1942).

(5) B. G. Zijnen, Van der Hegge, "Measurements of the velocity distribution in a plane turbulent jet of air," Appl. Sci. Res., Sect. A, Vol. 7, pp. 256-276 (1958).

(6) J. Mi, R. C. Deo and G. J. Nathan, "Characterization of turbulent jets from high-aspect-ratio rectangular nozzles," Phys. Fluids, Vol. 17, No. 6, pp. 068102.1-068102.4 (2005a).

(7) Z. D. Husain and A. K. M. F. Hussain, "Natural instability of free shear layers," AIAA Journal, Vol. 21, No. 11, pp. 1512 - 1517 (1983).

(8) T. Kiwata, S. Kimura, N. Komatsu, H. Murata and Y. H. Kim, "Flow characteristics of a plane jet with an extended lip-plate and serrated tabs," J. Fluid Science \& Technology, Vol. 4, No. 2, pp. 268-278 (2009).

(9) N. Rajaratnam, Turbulent Jets, Elsevier, Amsterdam (1976).

(10) D. Rockwell and E. Naudascher, "Self-sustaining Oscillations of flow past cavities," $J$. Fluids Engineering, Trans. ASME, Vol. 100, pp. 152-165 (1978).

(11) D. Rockwell and E. Naudascher, "Self-sustained oscillations of impinging free shear layers," Ann. Rev. Fluid Mech., Vol. 11, pp. 67 - 94 (1979). 